\title{
Communications
}

\section{Examen de spécialiste en vue de l'obtention du titre de spécialiste en Cytopathologie}

Lieu: Institut de Pathologie,

Université de Berne

\section{Date: 13 novembre 2018}

Délai d'inscription: toutes places déjà occupées, date prochaine: printemps 2019

Vous trouverez de plus amples informations sur le site web de l'ISFM www.siwf.ch $\rightarrow$ Domaines spécialisés $\rightarrow$ Titres de spécialiste et formations approfondies (formation postgraduée) $\rightarrow$ Pathologie

Examen en vue de l'obtention de la formation approfondie en psychiatrie et psychothérapie forensique à adjoindre au titre de spécialiste en psychiatrie et psychothérapie

Lieu: Universitäre Psychiatrische Kliniken (UPK), Wilhelm Klein-Strasse 27, 4012 Bâle

Date: le 3 décembre 2018

Délai d'inscription: le 31 octobre 2018

Vous trouverez de plus amples informations sur le site web de l'ISFM www.siwf.ch $\rightarrow$ Domaines spécialisés $\rightarrow$ Titres de spécialiste et formations approfondies (formation postgraduée) $\rightarrow$ psychiatrie et psychothérapie
Société Suisse de Psychiatrie et Psychothérapie de la Personne Agée

\section{Prix Jean Wertheimer 2019}

Le Professeur Jean Wertheimer (1933-1999) fut un pionnier dans le domaine de la psychiatrie de la personne âgée. En 1971, il fut appelé à l'Université de Lausanne pour y occuper la première chaire de psychiatrie de la personne âgée en Suisse. Il contribua à mettre en place une psychiatrie de la personne âgée reconnue dans le monde entier. Par la suite, il fut président de la Geriatric Psychiatry Section de l'OMS.

En son honneur, la Société Suisse de Psychiatrie et Psychothérapie de la Personne Agée a créé un prix d'encouragement pour récompenser des travaux prestigieux, des projets novateurs majeurs ou des objectifs éthiques dans l'esprit de Jean Wertheimer dans le domaine de la psychiatrie de la personne âgée en Suisse. Ce prix sera décerné tous les deux ans.

Les travaux et projets adéquats peuvent être envoyés d'ici le 30 novembre 2018.

\section{Règlement de concours Objectif}

Le prix récompense des travaux scientifiques de grande qualité et originalité dans la recherche de la psychiatrie de la personne âgée, des projets importants ou des objectifs éthiques. Les travaux soumis doivent se caractériser par leur aspect novateur et faire preuve d'une grande importance clinique.

Le prix Jean Wertheimer est attribué à une seule personne. La somme du prix s'élève à 5000 CHF. Le prix est remis au lauréat en personne lors de l'assemblée annuelle de la Société Suisse de Psychiatrie de la Personne Agée, qui aura lieu en juin 2019.

\section{Jury}

La Société Suisse de Psychiatrie et de Psychothérapie de la Personne Agée a nommé un jury qui décidera de l'attribution du prix avant fin février 2019.

Le jury est présidé par le Prof. Dr méd. Urs P. Mosimann, Berne, et comprend comme autres membres le Dr méd. Michel Gaillard, Lausanne, le Dr méd. Eva Krebs-Roubicek, Zurich, et le Dr méd. Nathalie Trächsel, Sion.

Le jury est libre et indépendant dans toutes ses décisions. Les décisions ne peuvent pas être contestées, la voie juridique est exclue. Les travaux ou candidatures qui n'ont pas été ou qui ne peuvent pas être retenus sont renvoyés à l'expéditeur sans justification, sans que cela implique un jugement positif ou négatif. L'attribution du prix peut être suspendue si les travaux soumis sont jugés insuffisants en qualité ou en quantité.

\section{Soumission}

Les travaux soumis doivent avoir été menés principalement en Suisse. Le lieu de travail de l'initiateur, de l'auteur principal ou du senior author doit se trouver en Suisse au moment de la soumission. Le travail peut être présenté en allemand, français, italien ou anglais.

Les documents suivants doivent accompagner la candidature:

- Curriculum vitae et liste des publications

- Lettre d'accompagnement présentant la pertinence du travail soumis

- Résumé du travail soumis d'une page A4 au maximum

- Travail original

Le travail ne doit pas déjà avoir reçu un autre prix scientifique ou avoir été sélectionné en vue d'un tel prix. Des participations parallèles à plusieurs concours doivent être annoncées lors de la soumission.

Les candidatures au prix Jean Wertheimer 2019 doivent être envoyées par écrit jusqu'au 30 novembre 2018 à:

Prof. Dr méd. Urs P. Mosimann Directeur de la Médecine

Insel Gruppe AG

Inselspital, Universitätsspital Bern

Friedbühlstrasse 53

3010 Berne

urspeter.mosimann[at]insel.ch www.inselgruppe.ch 
Société Suisse de Neuroradiologie

Médaille d'honneur en hommage à

Antonios Valavanis

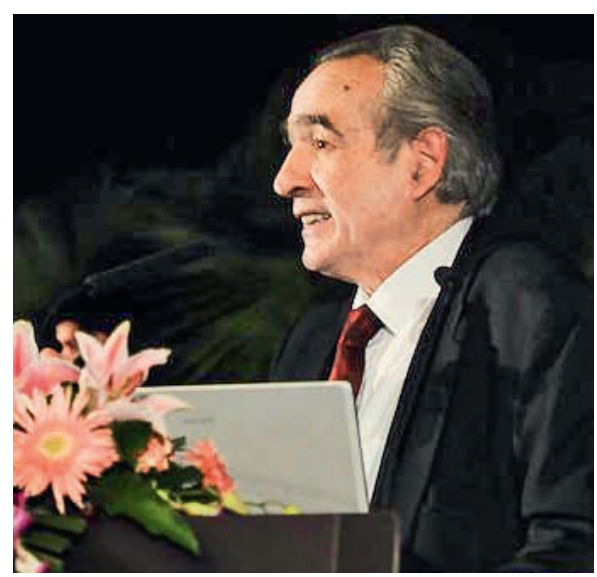

Antonios Valavanis

La Société Suisse de Neuroradiologie (SSNR) décide de décerner à l'avenir la médaille d'honneur Antonios Valavanis et de mettre en place un séminaire afin de saluer les accomplissements du Professeur Antonio Valavanis, ce grand Professeur et développeur de la neuroradiologie en Suisse.
La médaille d'honneur sera en principe décernée tous les 2 ans à partir de l'année 2019. Elle n'est pas mise au concours. Le lauréat doit être un excellent clinicien ayant un lien privilégié avec la Suisse, de préférence issu de l'espace européen et qui, grâce à ses accomplissements scientifiques et en tant qu'enseignant clinicien, défend et promeut l'ensemble de la neuroradiologie dans le même esprit que le Professeur Valavanis, et ce dans une mesure supérieure à la moyenne.

Antonios Valavanis ( $\left.{ }^{*} 1952\right)$ a étudié la médecine à l'université de Zurich et a ensuite suivi sa formation postgraduée au sein de l'hôpital universitaire de Zurich (USZ), où, d'abord en tant qu'élève puis en tant que collègue du Professeur Gazi Yasargil, neurochirurgien, il a été grandement impliqué dans la naissance de la neuroradiologie. Antonio Valavanis, un des tout premiers neuroradiologues, a créé l'institut puis la clinique de neuroradiologie au sein de l'USZ. Jusqu'à la fin de l'année 2017, il a été le directeur de cette clinique, et depuis 2014, il est président du comité du neurocentre clinique de l'hôpital universitaire de Zurich. De 1994 jusqu'à l'obtention du statut de Professeur émérite il y a quelques mois, Antonios Valavanis a en outre été le premier
Professeur ordinaire de neuroradiologie de l'université de Zurich.

Aux côtés de Peter Huber, Ernst Wilhelm Radü et Othmar Schubiger, Antonios Valavanis est un des membres fondateurs de la Société Suisse de Neuroradiologie (SSNR). Durant 15 ans, de 1993 à 1999 et de 2001 à 2010, Antonios Valavanis a été président de la SSNR. Lors de l'Assemblée générale 2018, Antonios Valavanis a été nommé membre honoraire de la SSNR.

Le Professeur Luca Remonda, Past-Président de la SSNR, déclare à propos de la médaille d'honneur: «Pratiquement personne n'a marqué de façon si décisive la neuroradiologie et même les neurosciences cliniques dans leur ensemble, tant au niveau professionnel que politique, à l'échelle nationale mais aussi internationale, qu'Antonios Valavanis. Avec cette médaille d'honneur, nous souhaitons rendre hommage à l'engagement inestimable, aux innombrables mérites et à l'œuvre de la vie d'Antonios Valavanis.»

Le conseil d'administration du prix se compose du président en fonction de la SSNR et de trois autres membres qui doivent être nommés par le comité de la SSNR et qui représentent la neuroradiologie clinique dans toute son étendue. 